\title{
Efficiency at rest: Magnetoencephalographic resting-state connectivity and individual differences in verbal working memory
}

\author{
David del Río , Pablo Cuesta , Ricardo Bajo , Javier García-Pacios , Ramón López-Higes , \\ Francisco del-Pozo , Fernando Maestú
}

\begin{abstract}
Inter-individual differences in cognitive performance are based on an efficient use of task-related brain resources. However, little is known yet on how these differences might be reflected on resting-state brain networks. Here we used Magnetoencephalography resting-state recordings to assess the relationship between a behavioral measurement of verbal working memory and functional connectivity as measured through Mutual Information. We studied theta $(4-8 \mathrm{~Hz})$, low alpha $(8-10 \mathrm{~Hz})$, high alpha $(10-13 \mathrm{~Hz})$, low beta $(13-18 \mathrm{~Hz})$ and high beta $(18-30 \mathrm{~Hz})$ frequency bands. A higher verbal working memory capacity was associated with a lower mutual information in the low alpha band, prominently among right-anterior and left-lateral sensors. The results suggest that an efficient brain organization in the domain of verbal working memory might be related to a lower resting-state functional connectivity across large-scale brain networks possibly involving right prefrontal and left perisylvian areas.
\end{abstract}

\section{Introduction}

Brain imaging research has revitalized interest in the neural basis of individual differences in cognitive skills (Newman and Just, 2005). In particular, results in the area tend to support the neural efficiency hypothesis, i.e. a reduction in task-related regional brain activity for gifted individuals, so that good performers consume less neural resources than bad performers for a given cognitive task (Grabner et al., 2004; Haier et al., 1988). However, recent findings regarding neural efficiency have not always been straightforward. For example, some studies have found that high performing subjects increase their recruitment of prefrontal brain areas during complex working memory processing to a higher extent than bad performers (Osaka et al., 2003, 2004). Task-related variables such as task demands, difficulty, and degree of subject's experience or familiarity with the task, modulate the relationship between brain activity and differences in cognitive performance (see Neubauer and Fink, 2009, for an extended review).

The neural adaptability concept (Prat and Just, 2011; Prat et al., 2007) might help explain these discrepant findings. High capacity subjects seem to recruit additional neural resources on an as-needed basis. Therefore, while good performers might show reduced regional brain activity at low/medium levels of task difficulty, they would also increase their recruitment of neural resources to a higher extent than poor performers with increased task difficulty. For example, Doppelmayr et al. (2005), using the Raven progressive matrices test, found that high performers, while working on easy items, displayed lower levels of brain activity when compared to bad performers (in agreement with the neural efficiency hypothesis). However, only good performers increased their brain activity when faced with increased task demands (neural adaptability).

In addition, functional connectivity (interaction between brain regions) has recently emerged as a crucial factor mediating the relationship between brain activity and cognition (Bressler and Tognoli, 2006; Uhlhaas and Singer, 2006; Varela et al., 2001). Therefore, recent studies have aimed to study how individual differences in cognitive skills are related not only to regional brain activity but also to functional connectivity patterns (e.g.: Prat and Just, 2011; Prat et al., 2007; Rypma et al., 2006). In particular, functional connectivity among prefrontal and posterior brain regions might add up critical evidence on the relationship between brain organization and cognitive skills, with prefrontal areas sustaining executive and working memory resources operating over posterior, task-related brain regions. However, divergent patterns of results have arisen. For example, Rypma et al. (2006) found that good task performers show a lower level of interplay between prefrontal and posterior brain areas, so that efficiency seemed to involve prefrontal resources to a lesser degree. In contrast, other studies have found higher levels of functional brain connectivity among prefrontal and other task-related areas in skilled participants (Osaka et al., 2003, 2004; Prat and Just, 2011; Prat et al., 2007). 
Again, task-related variables and neural adaptability may help explain the inconsistencies among different studies. While Rypma et al. (2006) used a relatively simple speeded processing task (the digit-symbol substitution task) other studies assessed functional brain connectivity during complex verbal working memory tasks. Therefore, an efficient use of functional brain connectivity might entail reduced functional coupling among executive prefrontal and other task-related brain areas at the lower levels of task difficulty, and enhanced levels of antero-posterior coupling when task demands are increased.

Hence, one outcome of neuroimaging studies about neural efficiency and cognitive performance is that results might depend on task difficulty. Yet, if individual differences are related to functional brain connectivity, we might expect neural efficiency signs to become observable not only during task performance, but also in the intrinsic connectivity pattern of resting-state activity. In fact, a wealth of data is highlighting the relevance of intrinsic connectivity networks, i.e., large-scale brain systems showing functional coupling during rest, to understand brain function (Bressler and Menon, 2010; Damoiseaux et al., 2006). The study of resting-state functional connectivity patterns and how they relate to individual differences in cognitive skills might thus complement studies about task-related functional connectivity and help define the basic signs of neural efficiency, without the confounding interference of task-related demands. Patterns of intrinsic functional coupling might reflect the integration and transfer of information across large-scale functional networks and thus provide important evidence on the efficiency of brain processing even in the absence of an explicit task.

Several previous results have found resting-state functional connectivity to be related to individual differences in cognitive performance, though the evidence is sparse. However, in a broad sense, these studies have emphasized a positive relationship between functional connectivity at rest and cognitive performance. Skilled performers tend to show increased levels of resting-state connectivity in comparison to lower performers (Hampson et al., 2006; Sala-Llonch et al., 2011; Song et al., 2008). These findings might seem at odds with the idea that a higher neural efficiency is related to a lower level of functional connectivity under low task demands. For example, Hampson et al. (2006) found functional coupling between core areas of the so called "Default-Mode Network" (DMN) to be positively related to working memory performance not only during task execution but also at rest. Similarly, Sala-Llonch et al. (2011) found that good performers in a working memory task showed greater connectivity in the precuneus/posterior cingulate node with respect to all other DMN nodes. Song et al. (2008) found that the individual Intelligence Quotient (IQ) as measured trough the Weschler Adult Intelligence Scale was positively related with an increased level of functional connectivity among bilateral dorsolateral prefrontal cortex and distributed brain areas at rest.

Notwithstanding, most of these studies have used fMRI BOLD responses to study intrinsic connectivity in functional brain networks. While providing an optimal reconstruction of brain activity, the BOLD signal becomes an indirect measurement of neural activity and we might also expect to obtain crucial evidence from resting-state Electroencephalography (EEG) and Magnetoencephalography (MEG) recordings measuring directly the temporal dynamics of brain oscillations arising from postsynaptic cortical currents.

Considering that oscillatory activity on different frequency bands is a hallmark of brain functioning, the study of functional connectivity through electromagnetic recordings might provide a unique window into how communication among brain networks is reflected on oscillatory brain activity (Sauseng and Klimesch, 2008). As with fMRI studies, evidence on the topic of how electrophysiological patterns of intrinsic brain connectivity are related to cognitive performance in non-pathological subjects is very scarce. However, recent evidence from EEG suggests that lower levels of electrophysiological brain coupling at rest may in some cases index neural efficiency. In particular,
Zhou et al. (2012) found that resting functional connectivity in the alpha and gamma bands correlated negatively with response speed in a subsequent go-no go task. According to these results, better performance is related to a lower long-range brain interplay at rest. Additionally, recent research has found a positive relationship between some network parameters obtained from resting-state MEG recordings putatively defining efficient connectivity and a composite index of performance across a variety of cognitive tests (Douw et al., 2011). Also van den Heuvel et al. (2009) reported a relationship between network parameters obtained from resting-state fMRI data and IQ.

Therefore, several resting fMRI and EEG/MEG studies show that intrinsic functional coupling might provide evidence about latent cognitive capacities and about the signatures of an efficient brain organization. The principal aim of the present work is to use MEG as a tool to study the relationship between resting-state functional connectivity and individual differences in cognitive skills. Specifically, and bearing in mind that prefrontal (top-down) regulation over task-related brain areas is crucial for executive attention, cognitive control and fluid abilities (Kane and Engle, 2002), we hypothesized that large-scale functional connectivity at rest, and particularly the interaction between prefrontal and posterior brain areas, might be related to individual differences in cognitive performance.

In addition, taking into account that the relationship between neural efficiency and cognitive performance might depend on specific functional brain networks related to the cognitive domain of interest (Grabner et al., 2004), we aimed to restrict to one well defined measure of cognitive proficiency in a restricted cognitive domain, in contrast to previous approaches which have used a variety of tasks in order to measure a wide spectra of cognitive abilities (Douw et al., 2011; van den Heuvel et al., 2009; Song et al., 2008). In particular, we focused on performance in the Reading Span Test (RST, Daneman and Carpenter, 1980 ), which is a widely used measure of cognitive proficiency in the domain of verbal WM. The RST is a classic representative example of so-called complex span tasks, where the subject is asked to simultaneously maintain and process information. Performance in complex span tasks has been found to be closely related to higher-level cognitive skills (Jarrold and Towse, 2006; Kane et al., 2004). The relationship of complex-span tasks with high order cognitive capacity stems from the high cognitive control demands imposed by the dual nature of the task (processing and storage, and continuous shifting between them) which are critical for a wide range of complex real world tasks (Engle, 2010). Complex span tasks such as the RST might thus constitute a suitable framework to study how connectivity between prefrontal and posterior brain areas is related to cognitive skills.

The RST measures a well defined area of cognitive processing, namely, verbal working memory (WM). The term verbal WM refers to the limited capacity system dedicated to the temporary storage of linguistic information, and to its simultaneous processing and manipulation (Just and Carpenter, 1992). In this regard, RST scores are good predictors of performance in complex linguistic tasks (Daneman and Carpenter, 1980; Daneman and Merikle, 1996; Just and Carpenter, 1992). While the study of a very circumscribed cognitive domain might hamper us from obtaining general conclusions about neural efficiency and "overall" cognitive processing, its main advantage is that we might relate our results to the functioning of specific large scale brain networks which are known to be related to this domain, such as left perisylvian and frontal executive networks (e.g. Osaka et al., 2003, 2004; Prat and Just, 2011; Prat et al., 2007).

Additionally, as suggested by an anonymous reviewer, the RST might also be related to the episodic buffer component proposed by Baddeley (2000). The episodic buffer is dedicated to form integrated units of information with time sequencing and to have links to long-term memory and semantic meaning. The memory retrieval part of the RST might pose a big burden on the episodic buffer as the information that the subject is asked to retain and the amount 
of time that the information should be retained clearly overrides the capacity of the phonological loop.

In this context, MEG, in comparison with EEG, provides a nonreference based measurement of brain activity that is not distorted by the various biological tissues between the cortex and the scalp, or by extracellular volume conduction currents (Stam et al., 2003). The study of functional connectivity based on electromagnetic brain recordings has gained considerable development over the last years, and different methods (each with its own advantages and disadvantages) have been proposed (see Pereda et al., 2005, for review). Among them, we aimed to use Mutual Information (MI) to estimate the functional coupling between MEG sensor pairs. Basically, MI reflects how much extra information one gets from one signal by knowing the outcomes of the other one, and one of its main advantages is that it provides a measurement of both linear and nonlinear influences between signal pairs (Hlaváčková-Schindler et al., 2007; Pereda et al., 2005). We expected interindividual differences in the RST to be related to long-distance resting functional connectivity as reflected by $\mathrm{MI}$, thus helping to delineate which patterns of resting-state organization characterize effective brain networks in the specific domain of verbal WM.

\section{Methods}

\subsection{Subjects}

The sample consisted of 20 right-handed volunteers ( 7 male, 13 female, mean age 26.30 years \pm 3.86 ). Subjects were all healthy university students recruited from the area of Madrid, with no neurological or psychiatric disorders according to self-report. All subjects were native Spanish speakers and signed a written informed consent to participate in the study. Five additional subjects of an original pool of 25 were discarded for analysis. Four of them were excluded because they failed to show a clear alpha peak in their power spectrum in the $8-13 \mathrm{~Hz}$ range. One additional participant was regarded as an outlier and excluded because of extremely high values of MI (above 3.4 SD from the mean of the group in the low alpha band). After removing this participant none of the remaining scored beyond $3 \mathrm{SD}$ from the mean of the group in any frequency band.

\subsection{Reading span task}

We used a modified Spanish version of the RST (Daneman and Carpenter, 1980) as a measure of verbal WM. The task consisted of sixty unrelated sentences ranging in length from 10 to 13 words. The content of half of the sentences was altered to create easily detectable semantic and/or pragmatic anomalies (e.g.: "according to the official historical report, the first person to land on the moon was a woman") while the other half was also easily identifiable as correct (e.g.: "as ice becomes hotter it is transformed into water"). Sentences were then randomly assigned to five different blocks consisting of three different sentence sets. Sentence sets contained two sentences in the first block and increased in one sentence as the subject progressed from one block to the next, up to a maximum set of six sentences.

Sentences were entered onto a computer running Superlab (Cedrus Corp., San Pedro, CA) and were displayed one at a time, with black letters on a white background, on the center of the computer's screen. Subjects were asked to read each sentence silently and perform a judgment about its acceptability by pressing the letter "a" on the computer's keyboard if they found the sentence to be acceptable or letter " $z$ " if they found the sentence to contain any semantic or pragmatic anomaly. Subjects were also instructed to try to remember the final sentence words across each sentence set and recall them aloud at the end of the set, when a red question mark appeared on the computer's screen. They were not asked to recall target words in the same order as they appeared, but were told not to begin with the target word of the last sentence in each trial. The experimenter annotated the subject's response to the recall part of the RST on a separate sheet. Before the session took place, subjects were allowed to have a small practice block consisting of four sentences similar to those used in the study, arranged into two different sets of size two.

\subsection{MEG recordings}

The MEG signal was measured trough a 148-channel whole-head magnetometer (Magnes 2500, 4-D Neuroimaging, Inc., San Diego, CA) housed in a magnetically shielded room. Subjects were asked to lie motionlessly on a bed with their head inside the helmet-like magnetometer, close their eyes and relax for approximately $5 \mathrm{~min}$. The signal was digitized with a $254.31 \mathrm{~Hz}$ sampling rate and filtered on-line between 0.1 and $50 \mathrm{~Hz}$. Thereafter, the signal was subjected to an adaptive filtering procedure that is part of the 4-D Neuroimaging software package in order to reduce the influence of environmental noise on the recordings. Magnetoencephalographic recordings were always carried out after the subject had completed the RST out of the MEG chamber.

\subsection{Mutual Information}

Mutual Information (MI) is based on the concept of Shannon entropy (Shannon, 1948), which is defined as the average amount of information gained from a measurement that specifies one particular value. Given $M$ possible outcomes $X_{i}$, each of them with probability $p_{i}$, the entropy is given by:

$I_{X}=-\sum_{i=1}^{M} p_{i} \log p_{i}$.

The MI (Hlaváčková-Schindler et al., 2007) between two signals is defined as:

$\mathrm{MI}_{X Y}=\sum P_{i j} \log \frac{P_{i j}}{P_{i} P_{j}}$

where $p i j$ is the joint probability of $X=X_{i}$ and $Y=Y_{j}$. MI is a measure of the information that $X$ and $Y$ share: how much information one gets from one signal by knowing the values of the other. Thus, $\mathrm{MI}=0,\left(P_{i j}=P_{i} P_{j}\right)$ for independent signals, whereas for identical signals the value of MI is the uncertainty contained in one of them alone.

Matlab version 7.13 (Mathworks, Natick, MA, USA) was used for the analysis with custom-written scripts. The first 1000 points of each record were dismissed. The remaining data was segmented into windows of $125+510+125$ samples per trial, corresponding to $0.5+2+0.5 \mathrm{~s}$ according to the current sampling rate $(254.31 \mathrm{~Hz})$. We only used the $2 \mathrm{~s}$ central segment and the first and the last 125 samples were used for filter border padding. MEG segments were visually inspected by an expert (D.R.) and trials containing artifacts were discarded. The average number of trials was $57 \pm 9$ (ranging between a minimum of 44 and a maximum of 82 ) per subject.

Data was subsequently filtered in the theta $(4-8 \mathrm{~Hz})$, low alpha $(8-10 \mathrm{~Hz})$, high alpha $(10-13 \mathrm{~Hz})$, low beta $(13-18 \mathrm{~Hz})$ and high beta $(18-30 \mathrm{~Hz})$ bands. We used an IIR Butterworth filter. The order of the filter was chosen as 6 , which corresponds to the maximum order and is consistent with the stability condition (i.e., all poles should be of the unit circle). Filtering was done on the segmented data with $0.5 \mathrm{~s}$ padding (as detailed above) on both sides of each segment in order to avoid the border effects.

The MI value across each possible sensor pair was then estimated over two-second time windows for all frequency bands. MI values for 
each one of these time windows were finally averaged to yield a single MI value for each sensor pair.

In an attempt to control spurious-random casual coupling, we used a surrogate data test (Schreiber and Schmitz, 2000) to cross-check statistical significance of observed MI values. Surrogate time series data was obtained from the original data by means of the iterative Amplitude Adjusted Fourier Transform (iAAFI) method that tries to preserve both power spectra as the histogram of the original time series (see Supplementary Fig. S1).

In order to evaluate the significance level, 50 surrogate data test per trial were calculated, i.e. each trial matrix ( 148 MEG sensors $\times 760$ time points) had 50 surrogate matrices, where each surrogate sensor's signal was obtained by iAAFT method from the original one. For all surrogate trials, MI was calculated in the same way as applied in the original data. Since the average number of trials was $57 \pm 9$ trials, $2850 \pm 450$ values of connectivity between each pair of sensors were obtained for each subject.

Next, we performed a random effects analysis to assess the signif( 148 sensors $\times 148$ sensors $\times 2000$ MI surrogate values $\times 20$ subjects) where the MI surrogate values corresponded with 50 surrogates $\times 40$ trials (the same number of trials was selected randomly from the available trials in each subject). From this data we calculated a $z$-score for each participant and channel pair according to the following equation:

$$
z_{i, j}=\frac{\mathrm{MI}_{i, j}-\left\langle\mathrm{MI}_{i, j}\right\rangle_{\text {surrogate }}}{\operatorname{std}\left(\mathrm{MI}_{i, j}\right)_{\text {surrogate }}} .
$$

Finally, a $t$ test compared z-values for each channel pair at the group level against zero (van Dijk et al., 2010) (see Supplementary Fig. S2). The results were considered significant at an uncorrected $\mathrm{p}$ value of 0.0001 . Only the survival significant connections passing the surrogate test were used for data analysis.

In order to reduce the dimensionality of the data, sensors were grouped in regions-of-interest (ROIs) composed by subsets of 12 neighboring channels. These ROIs roughly covered left anterior (la), right anterior (ra), left lateral (II), right lateral (rl), left central (Ic), right central ( $\mathrm{rc}$ ), left posterior (Ip) and right posterior (rp) areas of the scalp, as outlined in Fig. 1. They were outlined to approximate left and right frontal, temporal, parietal and occipital brain areas, respectively. These ROIs, we believe, reflect ongoing neurophysiological activity in frontal, temporal, parietal and occipital brain regions at a coarse level. A much better anatomical resolution may be achieved using source reconstruction, but in order to identify brain generators from MEG signals one has to solve the inverse problem, which is ill posed and sensitive to noise (see Bosboom et al., 2009; Castellanos et al., 2010 or Stam et al., 2006 for a similar approach).

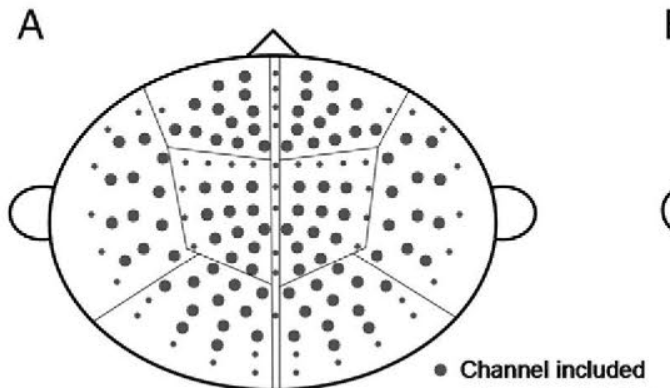

- Channel not included icance level of the resting functional connectivity. We made a matrix

Functional connectivity values between nearby MEG sensor pairs may be subject to field spread (Schoffelen and Gross, 2009). However, field spread effects are lower at widely spaced MEG sensors (Srinivasan et al., 2007). Hence, taking into account that we aimed to focus on large scale resting-state brain networks, we concentrate our analysis in long distance functional connectivity across each possible pair of sensor ROIs previously outlined (see Fig. 1). To do so, MI values between the sensor pairs composing each pair of ROIs were averaged and log transformed (only channel pairs where MI values passed the surrogate test were considered). The relationship between long distance functional connectivity and behavioral performance was then assessed through Pearson product-moment correlation tests between log transformed MI values and RST scores.

A permutation testing procedure was used to control for multiple comparisons at each frequency band (Nichols and Holmes, 2001). Two thousand surrogate correlation maps were calculated by randomly re-distributing RST scores among the participants. The highest absolute value of the Pearson correlation coefficient obtained for each surrogate was retained in order to obtain an empirical null distribution of the statistic. Statistical significant thresholds were obtained from the quantiles of the distribution of these values. For example, the 95th quantile is used for a p-value of 0.05 , which ensures that there is only a $5 \%$ chance that one or more correlation values from the original statistical map will present differences above threshold due to statistical fluctuations, and therefore corrects for multiple comparisons.

\section{Results}

\subsection{Reading span task}

Verbal WM capacity was scored as the total number of words that the subject was able to recall across the whole task. This measure has been shown to have higher reliability, distribute normally and show slightly higher correlations with reading comprehension tests than the usual score (maximum span) based on the size of the block where the subject is able to recall the whole word list in all trials (Friedman and Miyake, 2005). Moreover, a more continuous measure of verbal WM capacity is obtained by using this scoring method in comparison with the maximum span, which would ignore part of individual differences.

The RST scores based on total recall ranged between 19 and 56 words (from a total of 60 ) with a mean of $40.38 \pm 10.66$. We also calculated the classical maximum span score, based on the size of the block where the subject is able to recall the whole word list in all trials. Half a point was further added if the subject was able to recall the whole word list in at least one set of the next block (Daneman and Carpenter, 1980). Maximum span ranged between 2 and 5.5 with a mean of $3.62 \pm 1.20$. As in previous studies (Friedman and Miyake, 2005), the Pearson's product-moment correlation between both scoring methods was high $(r=0.846, p<0.001)$.

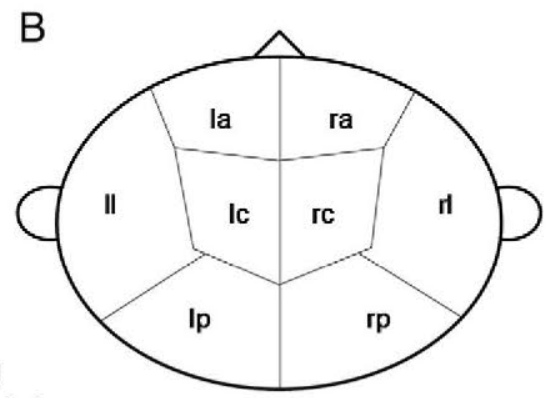

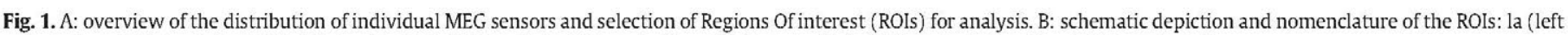
anterior), ra (right anterior), ll (left lateral), rl (right lateral), lc (left central), rc (right central), lp (left posterior) and rp (right posterior). 

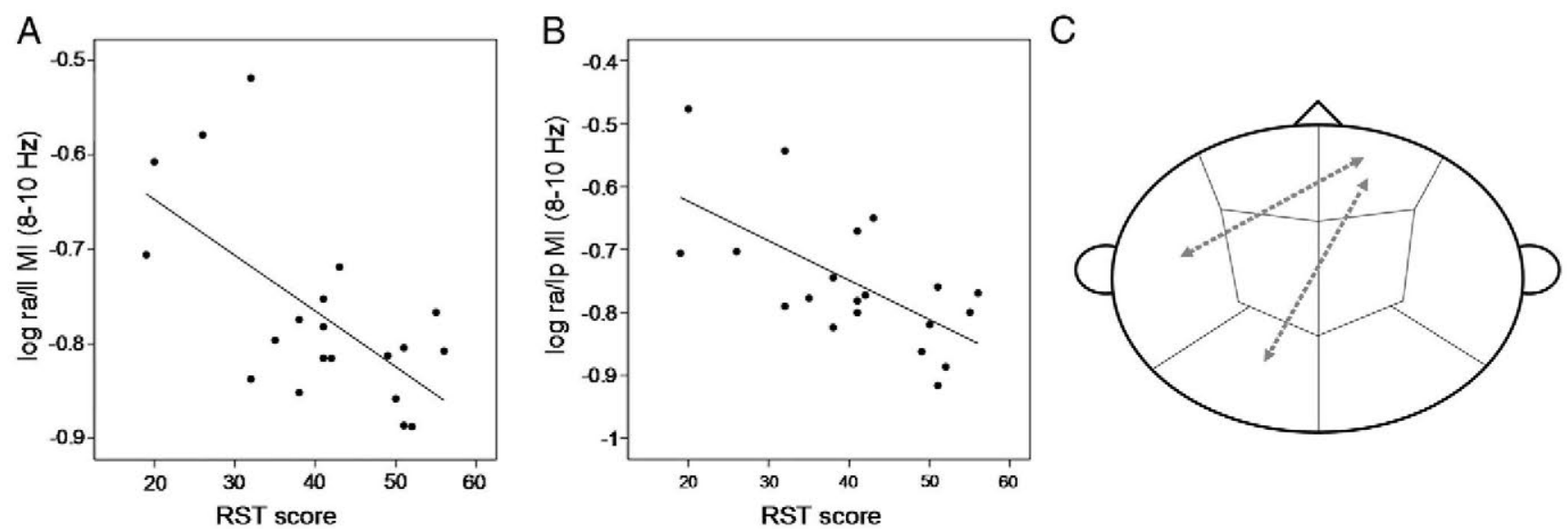

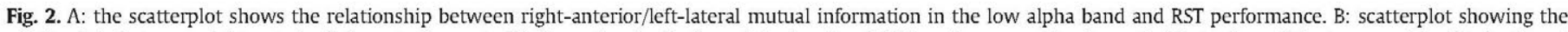

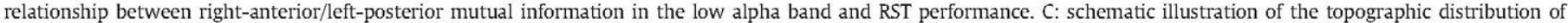
the results.

\subsection{Long distance functional connectivity (Mutual Information)}

No significant correlations between functional connectivity values and behavioral performance arise in the theta, high alpha, low beta or high beta frequency bands. However, in the low alpha band $(8-10 \mathrm{~Hz})$, significant highly negative Pearson correlation values were found between RST performance and right-anterior/left-lateral MI ( $r=-0.643$, $\left.p_{\text {corrected }}<0.05\right)$, as well as between RST performance and right-anterior/left-posterior MI $(r=-0.642$, p corrected $<0.05)$. As shown in Fig. 2, the lower the resting functional connectivity between each one of the pair of ROIs in the $8-10 \mathrm{~Hz}$ band, the higher the verbal WM performance of the subject. The correlations of the classical maximum span score with right-anterior/left-lateral and with rightanterior/left-posterior MI were also high $(\mathrm{r}=-0.592$, puncorrected $=$ 0.0060 , and $r=-0.596, p_{\text {uncorrected }}=0.0055$, respectively) although not significant after correction for multiple comparisons. As stated previously, the maximum span score ignores part of the individual variability in performance that the RST score based on the total recall captures (Friedman and Miyake, 2005). This might help explain the lower significance of the results.

Next, in order to estimate the relative contribution of right-anterior/ left-lateral and right-anterior/left-posterior MI to differences in RST scores, a step-wise multiple linear regression analysis was performed. RST scores were used as dependent variable and right-anterior/ left-lateral and right-anterior/left-posterior MI values were used as predictors. Results show that right-anterior/left-lateral MI accounted for $38 \%$ of variance in behavioral performance, without additional significant contribution from right-anterior/left-posterior MI.

In order to check whether our results in the lower alpha band are contaminated with a mutual source or not, we used an alternative measure of synchronization between pairs of signals: the phase lag index (PLI), which is not influenced by common underlying sources (Stam et al., 2007). Since the phase, as the imaginary part, measure absolute time synchronization, and assuming that a common source has zero lag, PLI between channels, where affected by a common source, should be zero. We calculated the PLI value for each pair of sensors (normalized between 0 and 1 ) and only pairs of sensors where PLI values were higher than 0.001 were considered.

For each subject, the MI values between pairs of sensors across previously defined ROIs were retained if they were not affected by common sources (i.e., with absolute PU values $>0.001$ ) and they passed the previously described surrogate test. Correlation values between RST performance and functional connectivity across right-anterior/left-lateral and right-anterior/left-posterior sensors were very similar to those obtained previously $\left(r=-0.633, p_{\text {corrected }}<0.05\right.$; and $r=-0.635$, $\mathrm{p}_{\text {corrected }}<0.05$, respectively). In addition, most of the sensor pairs across the ROIs where we obtained significant results seem to be unaffected by volume conduction on a subject by subject basis. The mean percentage of connections retained were $97.67 \%( \pm 1.80)$ for those involving right-anterior and left-lateral sensors and $97.92 \%( \pm 1.54)$ for those involving right-anterior and left-posterior sensors.

\section{Discussion}

The current study aims to outline resting-state brain signatures of efficient verbal WM processing. We used MEG to study the relationship between resting-state functional connectivity (measured trough MI) and verbal WM performance out of the MEG chamber. The RST was used as an index of verbal WM because of its capacity to tap simultaneously both the storage and the processing of linguistic material through the engagement of central executive resources (Daneman and Carpenter, 1980; Daneman and Merikle, 1996; Osaka et al., 2003, 2004). The present data indicate that patterns of resting connectivity might reflect functional aspects of brain organization related to cognitive capacity. More specifically, our results display an inverse relationship between functional connectivity across right anterior and left lateral/posterior MEG sensors in the low alpha band and verbal WM capacity.

Previous research has found resting-state connectivity patterns to be related to cognitive performance (Douw et al., 2011; Hampson et al., 2006; Sala-Llonch et al., 2011; Song et al., 2008; van den Heuvel et al., 2009). However, some of these previous studies have considered cognitive performance in a very broad sense, using composite measures of performance in different cognitive domains (Douw et al., 2011) or IQ measures (Song et al., 2008; van den Heuvel et al., 2009). Moreover, some of them have aimed to study the relationship between "small world" properties of the network (that is, a trend toward local clustering but overall integration; see for example Reijneveld et al., 2007) and overall cognitive efficiency (Douw et al., 2011; van den Heuvel et al., 2009). ${ }^{1}$ Hence, their results provide important insights on how general principles of network connectivity are related to efficient cognitive processing, but they give little information on the involvement of specific functional brain networks in specific cognitive functions. In contrast, we have aimed to address specifically the relationship between verbal WM capacity and resting functional connectivity.

\footnotetext{
Douw et al. (2011) provided additional data on the relationship between MEG sensor connectivity and some specific cognitive domains such as spatial memory, verba memory, processing speed and also WM (among others). However, in these analyses they focused exclusively on local connectivity (i.e., connectivity within nearby MEC sensors in pre-specified regions) instead of large-scale patterns of connectivity across extended areas.
} 
Some previous fMRI studies have highlighted positive relationships between WM performance and intrinsic connectivity within areas of the Default Mode Network (Hampson et al., 2006; Sala-Llonch et al., 2011). Notwithstanding, these previous studies used a different kind of task (the $n$-back paradigm) instead of the currently used RST, and there are important differences in what these different WM tasks might be measuring. In the $n$-back task the subject is required to decide whether a presented item (typically a letter) matches the one that appeared $n$ (usually 1 to 3 ) items ago. While this task imposes considerable demands on the continuous updating of memory sets, the RST is a kind of complex span task requiring the simultaneous processing and storage of verbal material, thus imposing continuous attention shifting between the processing and storage components of the task as well as high demands on linguistic processing. Moreover, RST demands serial recall, while $n$-back demands only recognition of the previously presented items (Kane et al., 2007). As stated in the introduction, performance on the RST may also partially rely on the episodic buffer component proposed by Baddeley (2000), devoted to the temporary maintenance of integrated information and its temporal sequencing. In particular the recall part of the RST would override the limits of the phonological buffer and depend on temporary episodic information. Hence, $n$-back and RST might measure partially distinct aspects of verbal WM. Crucially, although both n-back and complex span tasks such as the RST have been shown to explain individual differences in sentence memory (Roberts and Gibson, 2002) or fluid intelligence (Kane et al., 2007), they account for independent variance, suggesting that they do not reflect a single construct and are only loosely related.

When considering in detail our current results, one of the main findings attracting our attention is the relationship between verbal WM efficiency and long-distance alpha coupling. The timing of alpha oscillations shapes periods of increased and decreased neural excitabilities and a large amount of data suggests that it constitutes a powerful mechanism for the top-down control of perceptual and memory processes (Klimesch et al., 2007). In this regard, increased local alpha power has been suggested to reflect active inhibition of task-unrelated areas (Jensen and Mazaheri, 2010). Even more interesting for our current results are studies reporting long-range alpha synchronization (i.e., increased coupling between distant brain areas) related to information transfer and integration across extended large-scale brain networks (e.g. Doesburg et al., 2009; Palva et al., 2005, 2010). Furthermore, it has been proposed that, in comparison with locally connected neural assemblies, the communication across widespread extended brain networks would result prominently in frequencies of resonance in the theta or alpha range because of the transmission delay between neurons (von Stein and Sarnthein, 2000). Alpha (and theta) long-range synchronization might thus be particularly related to the engagement of large-scale networks involved in WM and attentional top-down control (Klimesch et al., 2007; Palva and Palva, 2007, 2011; Sauseng and Klimesch, 2008; von Stein and Sarnthein, 2000). Long-range MEG synchrony in the alpha band has been found to be enhanced during WM processing (Palva et al., 2005, 2010). Although similar increases are also found in the beta and gamma bands, alpha synchronization tend to be more prominent at the longest interareal distances (Palva et al., 2005), indicating that it participates in neural coupling across distant brain areas. During a visual WM task, Palva et al. (2010) found a slight trend for alpha synchronization to involve preferentially antero-posterior brain networks, while beta and gamma synchronizations tend to involve posterior cortical areas related to the binding of sensory representations. Also, language processing during reading has been found to be supported by a large-scale brain network displaying prominent synchronization in the alpha band (Kujala et al., 2007). Interareal coherence in the alpha band was displayed in a consistent manner across different subjects and stimulus presentation rates in this study, which reinforces the idea that alpha oscillations might serve to bind information across extended functional brain circuits.
Hence, the theta-alpha frequency range might set up a privileged channel to interchange information across extended brain networks, particularly those related to top-down executive control and WM processing. The topographic distribution of our results involving right anterior and left lateral and posterior MEG sensors (see Fig. 2) further indicates that verbal WM efficiency is related to long-range antero-posterior connectivity. In particular, the regression analysis shows that functional connectivity across right-anterior/left-lateral regions was the best predictor for verbal WM capacity (Table 1). The involvement of right anterior and left lateral sensors suggests that verbal WM efficiency might be related to the interplay of information between right frontal executive regions, which have been consistently related to attentional recruitment when a task becomes more demanding (Rypma and D'Esposito, 1999), and left perisylvian areas devoted to linguistic processing (Hickok and Poeppel, 2004). Previous research has shown how functional connectivity across extended networks involving executive fronto-parietal and linguistic perisylvian brain areas is related to verbal WM performance and complex language comprehension tasks (Osaka et al., 2004; Prat and Just, 2011; Prat et al., 2007).

Although task-related studies have usually emphasized higher levels of antero-posterior functional connectivity during the performance of complex tasks in skilled subjects (Osaka et al., 2004; Prat and Just, 2011; Prat et al., 2007), other works using relatively easy tasks have found high performers to show a lower level of synchronization between prefrontal and posterior brain areas (Rypma et al., 2006). These findings might be compatible with the idea of an efficient use of neural resources in high performing subjects, and with the idea of neural adaptability (Prat and Just, 2011; Prat et al., 2007) such that the interplay of information between prefrontal areas and other task-related regions is minimized under low task demands but can be increased on an as-needed basis. So, while good performers show a relative independence at rest between executive anterior brain areas involved in top-down control and left lateral and posterior regions involved in language processing, the higher interplay across large-scale antero-posterior networks in low performers might thus index a cost-inefficient brain organization in the domain of verbal WM. Low performers may be establishing a higher interplay between prefrontal executive and left hemisphere networks participating in verbal processing even when there is no task at hand and executive demands are minimal. In addition, these results are in agreement with recent EEG evidence showing a negative relationship between large-scale brain synchrony and processing speed (Zhou et al., 2012).

Finally, a possible limitation of the current study is the well known fact that correlations between nearby MEG and EEG sensors might be due to the influence of common sources (Schoffelen and Gross, 2009). However, field spread is expected to affect the interaction among nearby sensors, but less likely long distance MEG interactions as those reflected in our present results (Srinivasan et al., 2007). Moreover, to further contrast whether our results were likely to be contaminated by the presence of common sources, we calculated the PLI and results showed that only a very limited number of channel pairs were affected by common sources, providing additional support to our present results.

Table 1

Summary of the step-wise multiple linear regression analysis.

\begin{tabular}{|c|c|c|c|c|c|c|}
\hline \multicolumn{4}{|l|}{ Model } & \multicolumn{3}{|c|}{ Variables entered } \\
\hline Adjusted $\mathrm{R}^{2}$ & $\mathrm{~F}$ & df & p Value & Variable & Beta & p Value \\
\hline 0.380 & 12.652 & 1,18 & 0.002 & $\begin{array}{l}\text { Right-anterior/ } \\
\text { left-lateral MI }\end{array}$ & -0.643 & 0.002 \\
\hline
\end{tabular}




\section{Conclusions}

In summary, the current results suggest that neural efficiency might not only manifest itself in the task-related pattern of brain recruitment, but instead intrinsic functional connectivity and the resulting brain activity patterns could show signs of an efficient brain organization. Moreover, patterns of resting-state functional connectivity might reveal not only disruptions in brain processing related to cognitive disturbances (Uhlhaas and Singer, 2006), but also more subtle individual differences related to cognitive efficiency even in young healthy subjects. All in all, the results suggest that resting-state functional connectivity between right-anterior and left-lateral and posterior regions in the low alpha band reveal the efficiency of large-scale networks involved in verbal WM processes.

Supplementary data to this article can be found online at http:// dx.doi.org/10.1016/j.ijpsycho.2012.08.011.

\section{Acknowledgments}

Authors would like to thank Alberto Fernandez, Juan García-Prieto, Nazareth Castellanos and Asif Jamil for their invaluable help in the preparation of the manuscript. Pablo Cuesta is currently supported by the project PSI2009-14415-C03-01 of the Spanish Ministry of S\&T.

Baddeley, A., 2000. The episodic buffer: a new component of working memory? Trends in Cognitive Science 4, 417-423.

Bosboom, J.L.W., Stoffers, D., Wolters, E.C., Stam, C.J., Berendse, H.W., 2009. MEG resting state functional connectivity in Parkinson's disease related dementia. Journal of Neural Transmission 116, 193-202.

Bressler, S.L., Menon, V., 2010. Large-scale brain networks in cognition: emerging methods and principles. Trends in Cognitive Science 14, 277-290.

Bressler, S.L., Tognoli, E., 2006. Operational principles of neurocognitive networks. International Journal of Psychophysiology 60, 139-148.

Castellanos, N.P., Paúl, N., Ordóñez, V.E., Demuynck, O., Bajo, R., Campo, P., Bilbao, A., Ortiz, T., del-Pozo, F., Maestú, F., 2010. Reorganization of functional connectivity as a correlate of cognitive recovery in acquired brain injury. Brain 133 (Pt 8), 2365-2381.

Damoiseaux, J.S., Rombouts, S.A.R.B., Barkhof, F., Scheltens, P., Stam, C.J., Smith, S.M., Beckmann, C.F., 2006. Consistent resting-state networks across healthy subjects. Proceedings of the National Academy of Sciences of the United States of America $103,13848-13853$.

Daneman, M., Carpenter, P.A., 1980. Individual differences in working memory and reading. Journal of Verbal Learning and Verbal Behavior 19, 450-466.

Daneman, M., Merikle, P.M., 1996. Working memory and language comprehension: a meta-analysis. Psychonomic Bulletin \& Review 3 (4), 422-433.

Doesburg, S.M., Green, J.J., McDonald, J.J., Ward, L.M., 2009. From local inhibition to long-range integration: a functional dissociation of alpha-band synchronization across cortical scales in visuospatial attention. Brain Research 1303, 97-110.

Doppelmayr, M., Klimesch, W., Sauseng, P., Hödlmoser, K., Stadler, W., Hanslmayr, S., 2005. Intelligence related differences in EEG-bandpower. Neuroscience Letters $381,309-313$.

Douw, L., Schoonheim, M.M., Landi, D., van der Meer, M.L., Geurts, J.J.G., Reijneveld, J.C., Klein, M., Stam, C.J., 2011. Cognition is related to resting-state small-world network topology: an magnetoencephalographic study. Neuroscience 175, 169-177.

Engle, R.W., 2010. Role of working-memory capacity in cognitive control. Current Anthropology 51 (S1), 17-26.

Friedman, N.P., Miyake, A., 2005. Comparison of four scoring methods for the reading span test. Behavior Research Methods 37, 581-590.

Grabner, R.H., Fink, A., Stipacek, A., Neuper, C., Neubauer, A.C., 2004. Intelligence and working memory systems: evidence of neural efficiency in alpha band ERD. Cognitive Brain Research 20, 212-225.

Haier, R.J., Siegel, B.V., Nuechterlein, K.H., Hazlett, E., Wu, J.C., Paek, J., Browning, H.L., Buchsbaum, M.S., 1988. Cortical glucose metabolic rate correlates of abstract reasoning and attention studied with positron emission tomography. Intelligence $12,199-217$.

Hampson, M., Driesen, N.R., Skudlarski, P., Gore, J.C., Constable, R.T., 2006. Brain connectivity related to working memory performance. The Journal of Neuroscience 26, 13338-13343.

Hickok, G., Poeppel, D., 2004. Dorsal and ventral streams: a framework for understanding aspects of the functional anatomy of language. Cognition 92, 67-99.

Hlaváčková-Schindler, K., Paluš, M., Velmejka, M., Bhattacharya, J., 2007. Causality detection based on information-theoretic approaches in time series analysis. Physics Reports 441, 1-46.

Jarrold, C., Towse, J.N., 2006. Individual differences in working memory. Neuroscience 139, 39-50.
Jensen, O., Mazaheri, A., 2010. Shaping functional architecture by oscillatory alpha activity: gating by inhibition. Frontiers in Human Neuroscience 4, 186.

Just, M.A., Carpenter, P.A., 1992. A capacity theory of comprehension: individual differences in working memory. Psychological Review 99, 122-149.

Kane, M.j., Engle, R., 2002. The role of prefrontal cortex in working-memory capacity, executive attention, and general fluid intelligence: an individual differences perspective. Psychonomic Bulletin and Review 9, 637-671.

Kane, M.J., Hambrick, D.Z., Tuholski, S.W., Wilhelm, O., Payne, T.W., Engle, R.W., 2004 The generality of working memory capacity: a latent-variable approach to verbal and visuospatial memory span and reasoning. Journal of Experimental Psychology. General 133, 189-217.

Kane, M.J., Conway, A.R., Miura, T.K., Colflesh, G.J., 2007. Working memory, attention control and the n-back task: a question of construct validity. Journal of Experimental Psychology: Learning, Memory, and Cognition 33, 615-622.

Klimesch, W., Sauseng, P., Hanslmayr, S., 2007. EEG alpha oscillations: the inhibition timing hypothesis. Brain Research Reviews 53, 63-88.

Kujala, J., Pammer, K., Cornelissen, P., Roebroeck, A., Formisano, E., Salmelin, R., 2007. Phase coupling in a cerebro-cerebellar network at $8-13 \mathrm{~Hz}$ during reading. Cerebral Cortex 17, 1476-1485.

Neubauer, A.C., Fink, A., 2009. Intelligence and neural efficiency. Neuroscience and Biobehavioral Reviews 33, 1004-1023.

Newman, S.D., Just, M.A., 2005. The neural bases of intelligence: a perspective based on functional neuroimaging. In: Sternberg, R.J., Pretz, J. (Eds.), Cognition and Intelligence: Identifying the Mechanisms of the Mind. Cambridge University Press, New York pp. 88-103.

Nichols, T.E., Holmes, A.P., 2001. Nonparametric permutation tests for human neuroimaging: a primer with examples. Human Brain Mapping 15, 1-25.

Osaka, M., Osaka, N., Kondo, H., Morishita, M., Fukuyama, H., Aso, T., Shibasaki, H., 2003 The neural basis of individual differences in working memory capacity: an fMRI study. Neurolmage 18, 789-797.

Osaka, N., Osaka, M., Kondo, H., Morishita, M., Fukuyama, H., Shibasaki, H., 2004. The neural basis of executive function in working memory: an fMRI study based on individual differences. NeuroImage 21, 623-631.

Palva, S., Palva, J.M., 2007. New vistas for alpha-frequency band oscillations. Trends in Neurosciences $30,150-158$.

Palva, S., Palva, J.M., 2011. Functional roles of alpha band synchronization in local and large-scale cortical networks. Frontiers in Psychology 2, 204. http://dx.doi.org/ 10.3389/fpsyg.2011.00204

Palva, J.M., Palva, S., Kaila, K., 2005. Phase synchrony among neuronal oscillations in the human cortex. The Journal of Neuroscience 25, 3962-3972.

Palva, J.M., Monto, S., Kulashekhar, S., Palva, S., 2010. Neuronal synchrony reveals working memory networks and predicts individual memory capacity. Proceedings of the National Academy of Sciences of the United States of America 107, $7580-7585$.

Pereda, E., Quiroga, R.Q., Bhattacharya, J., 2005. Nonlinear multivariate analysis of neurophysiological signals. Progress in Neurobiology 77, 1-37.

Prat, C.S., Just, M.A., 2011. Exploring the neural dynamics underpinning individual differences in sentence comprehension. Cerebral Cortex 21, 1747-1760.

Prat, C.S., Keller, T.A., Just, M.A., 2007. Individual differences in sentence comprehension: a functional magnetic resonance imaging investigation of syntactic and lexical processing demands. Journal of Cognitive Neuroscience 19, 1950-1963.

Reijneveld, J.C., Ponten, S.C., Berendse, H.W., Stam, C.J., 2007. The application of graph theoretical analysis to complex networks in the brain. Clinical Neurophysiology $118,2317-2331$.

Roberts, R., Gibson, E., 2002. Individual differences in sentence memory. Journal of Psycholinguistic Research 31, 573-598.

Rypma, B., D'Esposito, M., 1999. The roles of prefrontal brain regions in components of working memory: effects of memory load and individual differences. Proceedings of the National Academy of Sciences of the United States of America 96, 6558-6563.

Rypma, B., Berger, J.S., Prabhakaran, V., Bly, B.M., Kimberg, D.Y., Biswal, B.B., D'Esposito, M., 2006. Neural correlates of cognitive efficiency. Neurolmage 33, 969-979.

Sala-Llonch, R., Peña-Gómez, C., Arenaza-Urquijo, E.M., Vidal-Piñeiro, D., Bargalló, N., Junqué, C., Bartrés-Faz, D., 2011. Cortex. http://dx.doi.org/10.1016/j.cortex.2011.07.006.

Sauseng, P., Klimesch, W., 2008. What does phase information of oscillatory brain activity tell us about cognitive processes? Neuroscience and Biobehavioral Reviews $32,1001-1013$

Schoffelen, J.-M., Gross, J., 2009. Source connectivity analysis with MEG and EEG Human Brain Mapping 30, 1857-1865.

Schreiber, T., Schmitz, A., 2000. Surrogate time series. Physica D 142, 346-382.

Shannon, C.E., 1948. A mathematical theory of communication. The Bell System Technical Journal 27 (379-423), 623-656.

Song, M., Zhou, Y., Li, J., Liu, Y., Tian, L., Yu, C., Jiang, T., 2008. Brain spontaneous functional connectivity and intelligence. NeuroImage 41, 1168-1176.

Srinivasan, R., Winter, W.R., Ding, J., Nunez, P.L., 2007. EEG and MEG coherence: measures of functional connectivity at distinct spatial scales of neocortical dynamics. Journal of Neuroscience Methods 166, 41-52.

Stam, C.J., Breakspear, M., van Cappellen van Walsum, A.M., van Dijk, B.W., 2003. Nonlinear synchronization in EEG and whole-head MEG recordings of healthy subjects. Human Brain Mapping 19, 63-78.

Stam, C.J., Jones, B.F., Manshanden, I., van Cappellen van Walsum, A.M., Montez, T., Verbunt, J.P.A., de Munck, J.C., van Dijk, B.W., Berendse, H.W., Scheltens, P., 2006. Magnetoencephalographic evaluation of resting-state functional connectivity in Alzheimer's disease. Neurolmage 32, 1335-1344.

Stam, C.J., Nolte, G., Daffertshofer, A., 2007. Phase lag index: assessment of functional connectivity from multi channel EEG and MEG with diminished bias from common sources. Human Brain Mapping 28, 1178-1193. 
Uhlhaas, P.J., Singer, W., 2006. Neural synchrony in brain disorders: relevance for cognitive dysfunctions and pathophysiology. Neuron 52 (1), 155-168.

van den Heuvel, M.P., Stam, C.J., Kahn, R.S., Hulshoff Pol, H.E., 2009. Efficiency of functional brain networks and intellectual performance. The Journal of Neuroscience 29, 7619-7624.

van Dijk, H., van der Werf, J., Mazaheri, A., Medendorp, W.P., Jensen, O., 2010. Modulations in oscillatory activity with amplitude asymmetry can produce cognitively relevant event-related responses. Proceedings of the National Academy of Sciences of the United States of America 107, 900-905.
Varela, F., Lachaux, J.-Philippe, Rodriguez, E., Martinerie, J., 2001. The brainweb: phase synchronization and large-scale integration. Nature Reviews. Neuroscience 2 , 229-239.

von Stein, A. Sarnthein, 2000. Different frequencies for different scales of cortical integration: from local gamma to long range alpha/theta synchronization. International Journal of Psychophysiology 38, 301-313.

Zhou, G., Liu, P., He, J., Dong, M., Yang, X., Hou, B., Von Deneen, K.M., Qin, W., Tian, J., 2012. Interindividual reaction time variability is related to resting-state network topology: an electroencephalogram study. Neuroscience 202, 276-282. 